\title{
EFFICACY, SAFETY AND TOLERABILITY OF AUGMENTATIVE rTMS IN TREATMENT OF MAJOR DEPRESSIVE DISORDER (MDD): A PROSPECTIVE COHORT STUDY IN CROATIA
}

\author{
Igor Filipcic $^{1,2,3}$, Zeljko Milovac ${ }^{1}$, Strahimir Sucic ${ }^{1}$, Tomislav Gajsak ${ }^{1}$, Ivona Simunovic Filipcic ${ }^{4}$, \\ Ena Ivezic ${ }^{1}$, Vjekoslav Aljinovic ${ }^{1}$, Ivana Orgulan ${ }^{1}$, Sandra Zecevic Penic ${ }^{1}$ \& Zarko Bajic ${ }^{5}$

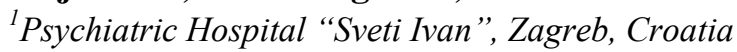 \\ ${ }^{2}$ Faculty of Medicine, Josip Juraj Strossmayer University of Osijek, Osijek, Croatia \\ ${ }^{3}$ School of Medicine, University of Zagreb, Zagreb, Croatia \\ ${ }^{4}$ Department of Psychological Medicine, University Hospital Center Zagreb, Zagreb, Croatia \\ ${ }^{5}$ Biometrika Healthcare Research, Zagreb, Croatia
}

received: 1.12.2016;

revised: 17.1.2017;

accepted: 23.1 .2017

\section{SUMMARY}

Background: An increasing body of research suggest that repetitive Transcranial Magnetic Stimulation (rTMS) is effective and safe treatment option for patients with major depressive disorder (MDD). The Psychiatric Hospital "Sveti Ivan "has the first TMS laboratory with rTMS and deep TMS (dTMS) in Croatia. The objective of this study was to assess the efficacy, safety and tolerability of augmentative rTMS treatment vs standard treatment in Croatian patients with major depressive disorder (MDD).

Subjects and methods: Total of 93 MDD patients were enrolled; 41 of them were treated by augmentative rTMS and 52 were treated by standard (psychopharmacotherapy and psychotherapy) therapy only. We delivered rTMS to the left dorsolateral prefrontal cortex at $120 \%$ motor threshold (10 Hz, 4-second train duration), 3000 pulses per session using a figure-eight coil, minimum of 20 sessions during four weeks. Our key outcome was the change in Hamilton Depression Scale (HAM-D17) result from baseline to $4^{\text {th }}$ week. Our secondary outcomes were changes in Hamilton Anxiety (HAM-A) and WHOQOL-BREF scales.

Results: After four weeks the changes of HAM-D17 and HAM-A results were significantly different between the group of patients treated by augmentative rTMS (48\% and 53\% decrease, respectively) and the group of patients treated by the standard therapy alone (24\% and 30\% decrease) $(P=0.004, P=0.007)$. Absolute benefit increase defined as the difference between rates of remission (HAM$D 17 \leq 7)$ in $r T M S$ and control group was 33\% $(P=0.001)$. Number of patients needed to treat with rTMS in order to achieve remission in one patient was $N N T=3$. In a group of patients treated with augmentative rTMS 21/41 (51\%), and in control group $17 / 52$ (33\%) were responders $(P=0.071)$.

Conclusions: It seems that augmentative treatment with rTMS is more effective on depression and anxiety symptoms than standard therapy in MDD with equal safety and tolerability. Randomized, controlled studies are required to verify this finding.

Key words: repetitive transcranial magnetic stimulation (rTMS) - major depressive disorder (MDD) - anxiety

\section{INTRODUCTION}

Depression is now the leading cause of disability worldwide and it is a major contributor to the overall global burden of disease (WHO 2016). Clinical response cannot be achieved to antidepressant pharmacotherapy or psychotherapy in more than $30 \%$ of patients (Rush et al. 2003). Patients which have been diagnosed with a major depressive disorder (MDD), but who fail to experience sufficient response after adequate rounds of pharmacotherapy or psychotherapy are called treatmentresistant depression (TRD) patients.

With the development of neuroscience, technology and psychiatry, we are now able to treat TRDs with repetitive transcranial magnetic stimulation (rTMS). It is safe and efficacious, noninvasive brain stimulation treatment for medication resistant MDD (Liu et al. 2014). Repetitive transcranial magnetic stimulation (rTMS) is a neurostimulation and neuromodulation technique. It is based on the principle of electro- magnetic induction. The TMS coil device modulates the electrical activity of the brain using a magnetic field. Depressive disorder rTMS treatment usually involves one of three protocols: high frequencies rTMS (HF rTMS) protocol, which is applied to the left dorsolateral prefrontal cortex (DLPFC); low frequency rTMS (LF rTMS) protocol applied to the right DLPFC and bilateral HF / LF rTMS.

The selection of DLPFC as a cortical target for rTMS application is based on pathophysiological changes. Functional images of the brain in depressed patients showed a decrease in cortical blood flow, glucose and oxygen consumption in the left frontal region (Kennedy et al. 1997), reflecting a hypometabolic state, accompanied by hypermetabolism in the right prefrontal areas (Bench et al. 1995). DLPFC is synaptically connected to the limbic system which takes part in the regulation of mood (striatum, thalamus and anterior cingulate cortex) (Paus et al. 2001). It is believed that rTMS over the DLPFC modulates brain network involved in the 
regulation of mood and affects various neurotransmitters. Lan and colleagues (2016) described the structural changes in the brain (gray matter volume increased by $3.5-11.2 \%$ ) in patients with a depressive disorder after rTMS treatment (Lan et al. 2016). Four regions in which they detect these changes were: left anterior cingulate cortex, the left insula, left superior temporal gyrus and the right angular gyrus. Increase in the volume of the left anterior cingulate cortex correlated with improvement of depression severity (Lan et al. 2016).

The first attempts at treating depressive disorder with rTMS are shown in a study by George and colleagues in 1995 after which numerous papers were published on this topic (George et al. 1995). A large number of randomized controlled trials (RCTs) and meta-analyses supported the antidepressant effect of TMS in patients with MDD when it was used in daily sessions over several weeks directed to the dorsolateral prefrontal cortex (DLPFC). Two large, multicenter, randomized controlled trials showed superior antidepressant efficacy of rTMS over sham rTMS. In the study by O'Reardon and colleagues, in 2007, 301 patients with TRD were treated with placebo or active rTMS over the left frontal cortex (DLPFC). After 6 weeks, patients in the active TMS group were about twice as likely to achieve remission compared to patients in the placebo group (MADRS: $14.2 \%$ versus $5.2 \%$, HAMD $17: 15.5 \%$ versus $7.1 \%$, HAMD 24 , $17.4 \%$ versus $8.2 \%$ ). Antidepressant effects were greater in TRD patients with less medication resistance (O'Reardon et al. 2007). In 2008, with the support of this and other similar research FDA (Food and drug administration) approved rTMS device for clinical treatment of TRDs. Another multicenter study involving a larger number of patients was the study by George and colleagues in 2010. It was conducted on 190 patients with MDD who were not taking their medications. A protocol of $10 \mathrm{~Hz}$ in the left prefrontal cortex with motor threshold to $120 \%$ (3000 pulses per session) was applied through three weeks of daily treatment. Remission was achieved in $14.1 \%$ of patients of the active group compared to $5.1 \%$ in the placebo group (George et al. 2010).

In a meta-analysis which studied the clinical response, remission, and the rate of discontinuation of therapy during treatment with HF rTMS, data were involved from 29 randomized controlled trials with a total of 1371 patients (Berlim et al. 2014). After an average of 13 sessions, clinical response was $29.3 \%$ and remission $18.6 \%$, three times more than among those who received placebo therapy (Berlim et al. 2014).

The Psychiatric Hospital "Sveti Ivan" is the first hospital in Croatia to perform rTMS in therapeutic purposes. The objective of this study was to examine the efficacy, safety and tolerability of HF rTMS in treatment of MDD in Croatian patients.

\section{SUBJECTS AND METHODS}

\section{Study design}

This prospective cohort study was done during 2016 at Psychiatric Hospital Sveti Ivan, Zagreb, Croatia. The study protocol was approved by the Ethics Committee of Psychiatric Hospital Sveti Ivan. Informed consent was obtained from all patients. The study complied with World Medical Association Declaration of Helsinki 2013 (World Medical Association 2013).

\section{Subjects}

The targeted population was patients of both sexes, diagnosed with major depressive disorder (ICD-10: F32 and F33) who were treated in a psychiatric hospital as outpatients, in daily hospital or who were hospitalized. Inclusion criteria were: ICD-10 and DSM-V major depression disorder, single or recurrent episode, treatment resistant depression (according to the Croatian clinical guidelines for treatment of depressive disorder, Mihaljevic et al. 2013) and ages between 20 and 70 . Exclusion criteria were previous treatment with TMS, feromagnetic material close to the head, cardiac pacemaker, and implanted electronic device, history of uncontrolled epilepsy, suicidal ideation. We chose a consecutive sample of patients by the order of their arrival at the exam during the enrollment period.

\section{Needed sample size}

Power analysis was performed before the start of the enrollment. A sample size of 68 achieves $80 \%$ power at $\mathrm{p}<0.05$ to detect a standardized effect size $\mathrm{f} \geq 0.20$ which corresponds to partial eta squared of $\eta^{2}=0.04$ of the repeated measures analysis of variance within-between interaction. Expecting up to $15 \%$ of respondents lost to follow up, the initially needed sample size was determined to be $n=80$ with equal sizes of two study groups. Power analysis was done in PASS 14 Power Analysis and Sample Size Software (2015) (NCSS, LLC, Kaysville, Utah, USA).

\section{Outcomes}

Our key outcome was the change in Hamilton Depression Scale (HAM-D17) result from baseline to $4^{\text {th }}$ week. HAM-D17 is a gold standard measure of the severity of depressive symptoms (Hamilton 1960, Bodo et al. 2016). Our secondary outcomes were the proportion of patients experiencing remission of symptoms defined as HAM-D17 score $\leq 7$, proportion of patients experiencing a positive response to treatment defined as the reduction in total score of $>50 \%$ (Cusin et al. 2009), changes in results of Hamilton Anxiety Scale (HAMA14) (Hamilton 1959) and four dimensions of The World Health Organization Quality of Life questionnaire (WHOQOL-BREF) (World Health Organization 
1993). HAM-A14 is originally clinician based questionnaire (Thomson 2015). The four WHOQOL-BREF dimensions were: physical health (items 3,4,10,15, 16,17,18), psychological health (items 5,6,7,11,19,26), social relationship (items 20,21,22) and environment (items 8,9,12,13,14,23,24,25) (World Health Organization 1996). HAM-D17 and HAM-A14 were administered by experienced and trained clinical rates, psychiatrists at baseline and after four weeks. WHOQOL-BREF was administered by patients themselves.

\section{Independent variable}

Our independent variable was treatment with augmentative rTMS and standard therapy versus treatment with standard therapy (psychopharmacotherapy and psychotherapy) alone. Patients were allocated in the study groups accordingly to their subjective preferences and physician estimated tolerability based on initial mapping procedure. We delivered rTMS to the left dorsolateral prefrontal cortex at $120 \%$ motor threshold (10 Hz, 4-second train duration), 3000 pulses per session for a minimum of 4 weeks using a figure-eight coil. Each patient received a minimum of 20 sessions. All patients continued taking psychopharmacotherapy during their HF rTMS treatment.

\section{Possible confounders}

Possible confounders whose effect we tried to control by multivariate analysis were sex, age, education, marital status, work status, diagnosis, existence of psychotic symptoms, duration of primary psychiatric illness, hospitalization or treatment in daily hospital or outpatients, severity of MDD at enrollment measured by the Clinical Global Impression-Severity (CGI-S) scale, number of previous psychiatric hospitalizations, number of psychiatric comorbidities, and number of somatic illnesses.

\section{Statistical analysis}

The level of statistical significance was set at $\mathrm{p}<0.05$, and we gave all confidence intervals at $95 \%$ level. In all instances, we used two-tailed tests. Differences between baseline measurement and the measurement at $4^{\text {th }}$ week follow-up were expressed as absolute and relative changes. The main statistical data analysis was done by analysis of covariance. Normality of univariate distributions were tested by Shapiro-Wilk test, and analysis of skewness and kurtosis. Outliers were defined as the cases with standardized values $\mathrm{z}>2.58$. As the sensitivity analysis we repeated the analysis with and without removal of outliers. Homogeneity of variances was tested by Levene's test. Age, education, marital status, work status, diagnosis, psychotic symptoms, mode of treatment, number of psychiatric hospitalizations, Clinical global impression severity (CGI-S), number of psychiatric comorbidities, and number of somatic illnesses were controlled as covariates. Partial eta squared $\left(\eta^{2}\right)$ was given as the standardized effect size. Statistical data analysis was done by NCSS 10 Statistical Software (2015) (NCSS, LLC. Kaysville, Utah, USA).

\section{RESULTS}

Total sample of 93 patients were enrolled; 41 of them allocated to the rTMS treatment, and 52 allocated to the standard treatment (Figure 1). Two study groups were comparable in terms of sex, age, work status, diagnosis, existence of psychotic symptoms and psychotropic medication (Table 1). However, there were several potentially clinically relevant differences between two groups that we tried to control by multivariate analysis. Patients in rTMS group were better educated, more often in a relationship, with somewhat longer duration of primary psychiatric illness and less psychiatric comorbidities.

\section{Assumptions of ANCOVA}

Hamilton Depression Scale at baseline and at $4^{\text {th }}$ week in both group did not significantly deviate from the theoretically expected normal distribution (in rTMS group: Shapiro-Wilk statistic $=0.99, \mathrm{df}=40, \mathrm{p}=0.851$; in control group: Shapiro-Wilk statistic $=0.97, \mathrm{df}=49$, $\mathrm{p}=0.281$ ). Only the distribution of HAM-D17 in rTMS group at $4^{\text {th }}$ week follow up may be considered moderately skewed $(0.56)$. Other three distributions were approximately symmetrical with skewness $<0.32$. At baseline, mean and median were the same in control group and irrelevantly different in rTMS group (mean=17, median=18) what indicates that distributions were symmetrical. At $4^{\text {th }}$ week follow up, mean and median were the same in control group, and irrelevantly different in rTMS group (mean=9, median=8). In control group we detected $2(3.8 \%)$ outliers in the baseline HAM-D17 measurement with $\mathrm{z}>2.58$, and 1 $(2.0 \%)$ in $4^{\text {th }}$ week follow-up. In HAM-A14, in control group, we detected $1(2.2 \%)$ outlier at baseline and 2 $(4.3 \%)$ at $4^{\text {th }}$ week follow-up. All secondary outcomes were approximately normally distributed except HAMA14 in rTMS group. Skewness of this distribution was 1.09 , and kurtosis was 0.30 . In all cases Levene's test of equality of error variances did not indicate significant heterogeneity.

\section{Main results}

After four weeks, Hamilton Depression Scale and Hamilton Anxiety Scale results were statistically significantly different between the group of patients treated by rTMS and standard therapy (48\% and 53\% decrease, respectively) and the control group treated by the standard therapy only ( $24 \%$ and $30 \%$ decrease), after adjustment for HAM-D17, HAM-A14 and WHOQOL-BREF 
Table 1. Participants' baseline characteristics and psychotropic medication used during the trial

\begin{tabular}{|c|c|c|c|c|}
\hline & \multicolumn{2}{|c|}{ Control $(\mathrm{n}=52)$} & \multicolumn{2}{|c|}{ rTMS $(n=41)$} \\
\hline & & & & \\
\hline male & 18 & $(34.6)$ & 15 & $(36.6)$ \\
\hline female & 34 & $(65.4)$ & 26 & $(53.4)$ \\
\hline Age (years), median (IQR) & 55 & $(49-61)$ & 53 & $(42-61)$ \\
\hline \multicolumn{5}{|l|}{ Education } \\
\hline primary & 5 & (9.6) & 1 & (2.4) \\
\hline secondary & 37 & $(71.2)$ & 25 & $(61.0)$ \\
\hline university & 10 & (19.2) & 15 & $(36.6)$ \\
\hline \multicolumn{5}{|l|}{ Marital status } \\
\hline in a relationship & 26 & $(50.0)$ & 24 & $(58.5)$ \\
\hline single & 26 & $(50.0)$ & 17 & $(41.5)$ \\
\hline \multicolumn{5}{|l|}{ Work status } \\
\hline employed & 24 & $(46.2)$ & 16 & $(39.0)$ \\
\hline unemployed & 9 & (17.3) & 12 & (29.3) \\
\hline retired & 19 & $(36.5)$ & 13 & $(31.7)$ \\
\hline \multicolumn{5}{|l|}{ CLINICAL CHARACTERISTICS } \\
\hline \multicolumn{5}{|l|}{ Diagnosis } \\
\hline depressive episode (F32) & 9 & $(17.3)$ & 9 & $(22.0)$ \\
\hline recurrent depressive disorder (F33) & 43 & (82.7) & 32 & $(78.0)$ \\
\hline Psychotic symptoms & 10 & $(19.2)$ & 8 & $(19.5)$ \\
\hline Duration of illness (years), median (IQR)* & 3 & $(1-10)$ & 5 & $(0-11)$ \\
\hline \multicolumn{5}{|l|}{ Mode of treatment } \\
\hline outpatients & 0 & $(0.0)$ & 21 & $(51.2)$ \\
\hline daily hospital & 12 & (23.1) & 3 & $(7.3)$ \\
\hline hospitalized & 40 & (76.9) & 17 & $(41.5)$ \\
\hline Number of psychiatric hospitalizations, median (IQR) & 2 & $(1-6)$ & 3 & $(1-7)$ \\
\hline \multicolumn{5}{|l|}{ Clinical global impression - severity (CGI-S) } \\
\hline up to moderately ill & 31 & $(59.6)$ & 21 & $(51.2)$ \\
\hline significantly ill & 13 & $(25.0)$ & 18 & (43.9) \\
\hline severely or very severely ill & 8 & (15.4) & 2 & $(4.9)$ \\
\hline \multicolumn{5}{|l|}{ Number of psychiatric comorbidities } \\
\hline none & 14 & (26.9) & 10 & (24.4) \\
\hline one & 23 & $(44.2)$ & 26 & $(63.4)$ \\
\hline two or more & 15 & $(28.8)$ & 5 & $(12.2)$ \\
\hline \multicolumn{5}{|l|}{ Number of somatic illnesses } \\
\hline none & 19 & $(36.5)$ & 16 & $(39.0)$ \\
\hline one & 12 & (23.1) & 9 & $(22.0)$ \\
\hline two & 6 & $(11.5)$ & 9 & $(22.0)$ \\
\hline three or more & 15 & $(28.8)$ & 7 & (17.1) \\
\hline \multicolumn{5}{|l|}{ PSYCHOTROPIC MEDICATION } \\
\hline Anxiolytics & 41 & $(78.8)$ & 31 & (75.6) \\
\hline SSRI & 16 & $(30.7)$ & 12 & $(29.2)$ \\
\hline SNRI & 20 & $(38.4)$ & 14 & $(34.1)$ \\
\hline NaSSA & 19 & $(36.5)$ & 9 & $(21.9)$ \\
\hline DNRI & 3 & $(5.7)$ & 2 & $(4.8)$ \\
\hline SARI & 6 & $(11.5)$ & 3 & $(7.3)$ \\
\hline Other antidepressants & 6 & (11.5) & 6 & $(14.6)$ \\
\hline Overall $2^{\text {nd }}$ generation antidepressants & 38 & $(73.0)$ & 34 & $(82.9)$ \\
\hline
\end{tabular}

Data are presented as number (percentage) of participants if not stated otherwise

Abbreviations: $\mathrm{IQR}=$ interquartile range; $\mathrm{SSRI}=$ selective serotonin reuptake inhibitors;

SNRI = serotonin and norepinephrine reuptake inhibitors; NaSSA = noradrenergic and specific serotonergic antidepressants;

DNRI = norepinephrine-dopamine reuptake inhibitors; SARI = serotonin antagonist and reuptake inhibitors

* Duration of primary psychiatric diagnosis 
baseline values as well as for patients age, education, marital status, work status, diagnosis, psychotic symptoms, mode of treatment, number of psychiatric hospitalizations, Clinical global impression - severity (CGI-S), number of psychiatric comorbidities, and number of somatic illnesses (Table 2). Proportion of patients achieving remission defined as HAM-D17 $\leq 7$ points was significantly and clinically relevantly higher in rTMS than in control group (Table 2). Absolute benefit increase defined as the difference between rates of remission (HAM-D17 $\leq 7)$ in rTMS and control group was $\mathrm{ABI}=33 \%$. Relative benefit increase was $\mathrm{RBI}=201 \%$. Number of patients needed to treat with rTMS in order to achieve remission in one patient was $\mathrm{NNT}=3$. In a group of patients treated with augmentative rTMS $21 / 41(51 \%)$, and in control group 17/52 $(33 \%)$ were responders (a decrease in HAM-D scores of at least $50 \%)(\mathrm{P}=0.071)$. We have not observed significant differences in changes of WHOQOL-BREF four dimensions.

Table 2. Differences in depression, anxiety and quality of life indicators from baseline to 4 weeks later

\begin{tabular}{|c|c|c|c|c|c|c|c|c|c|c|c|c|c|c|}
\hline \multirow{3}{*}{ HAM-D17 } & \multicolumn{6}{|c|}{ Control (n=52) } & \multicolumn{4}{|c|}{ rTMS $(n=41)$} & \multirow{3}{*}{$\frac{\Delta}{-8.1}$} & \multirow{3}{*}{$\begin{array}{l}\Delta \% \\
48 \%\end{array}$} & \multirow{3}{*}{$\frac{P}{0.004}$} & \multirow{3}{*}{$\begin{array}{c}\text { Effect } \\
0.10\end{array}$} \\
\hline & \multicolumn{2}{|c|}{ Baseline } & \multicolumn{2}{|c|}{ After 4 weeks } & \multirow{2}{*}{$\begin{array}{c}\Delta \\
-4.9\end{array}$} & \multirow{2}{*}{$\begin{array}{l}\Delta \% \\
24 \%\end{array}$} & \multicolumn{2}{|c|}{ Baseline } & \multicolumn{2}{|c|}{ After 4 weeks } & & & & \\
\hline & 18 & $(6.8)$ & 13 & $(6.5)$ & & & 17 & $(5.7)$ & 9 & $(6.0)$ & & & & \\
\hline Remission $(\leq 7), 1$ & 4 & (7.7) & 12 & $(23.1)$ & 15.4 & $200 \%$ & 1 & (2.4) & 20 & $(48$. & 46.4 & $1933 \%$ & 0.001 & 0.13 \\
\hline Resp & & & 17 & $(32.7)$ & & & & & & 2) & 18.5 & $57 \%$ & 0.071 & 0.19 \\
\hline HAI & 22 & $(10.8)$ & 15 & $(8.0)$ & -6.7 & $30 \%$ & 21 & (8.6) & 10 & $(7.7)$ & -11.3 & $53 \%$ & 0.007 & 0.09 \\
\hline & & & & & & & & & & & & & & \\
\hline & 46 & (19.4) & 43 & $(25.1)$ & -3.1 & $7 \%$ & 40 & $(16.6)$ & 43 & & 2.5 & $6 \%$ & 0.504 & 0.01 \\
\hline & 44 & $(21.6)$ & 49 & $(18.3$ & 5.0 & $11 \%$ & 36 & (19.7) & 4 & $(20$ & 9.0 & $25 \%$ & 0.872 & 0.00 \\
\hline Social & 49 & (24.3) & 52 & $(23.7)$ & 3.2 & $6 \%$ & 46 & $(23.5)$ & 50 & $(21.2)$ & 3.4 & $7 \%$ & 0.423 & 0.01 \\
\hline Environment & 57 & $(17.8)$ & 62 & $(17.6)$ & 5.4 & $9 \%$ & 61 & $(15.0)$ & 65 & $(17.5)$ & 3.8 & $6 \%$ & 0.276 & 0.02 \\
\hline
\end{tabular}

Data are presented as mean (standard deviation) if not stated otherwise

Abbreviations: HAM-D17 = Hamilton Depression Scale; HAM-A14 = Hamilton Anxiety Scale; WHO BREF $=$ World

Health Organization Quality of Life Brief Questionnaire dimensions; $\Delta=$ absolute difference between the baseline and measurement after 4 weeks; $\Delta \%=$ relative difference calculated as absolute difference divided by the baseline value; $\mathrm{P}=$ analysis of covariance, statistical significance of the difference at $4^{\text {th }}$ week follow up adjusted for baseline values of all outcomes and for age, education, marital status, work status, diagnosis, psychotic symptoms, mode of treatment, number of psychiatric hospitalizations; for Remission and Response Pearson Chi-Square; Clinical global impression - severity (CGI-S), number of psychiatric comorbidities, number of somatic illnesses, Effect $=$ partial eta squared $\left(\eta^{2}\right)$ given as the standardized effect size for numeric variables; Cramer's V given for categorical variables

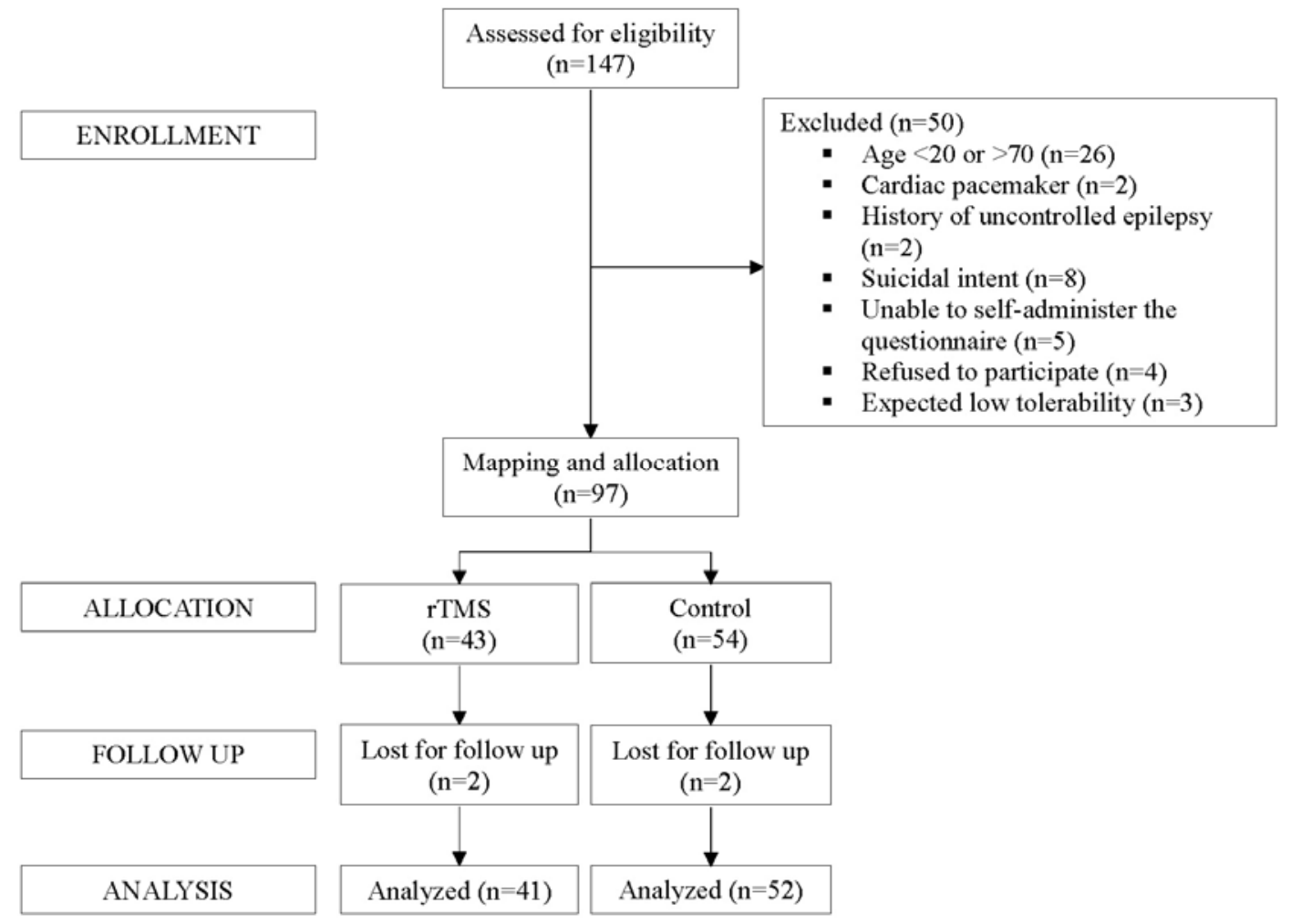

Figure 1. Study flow 


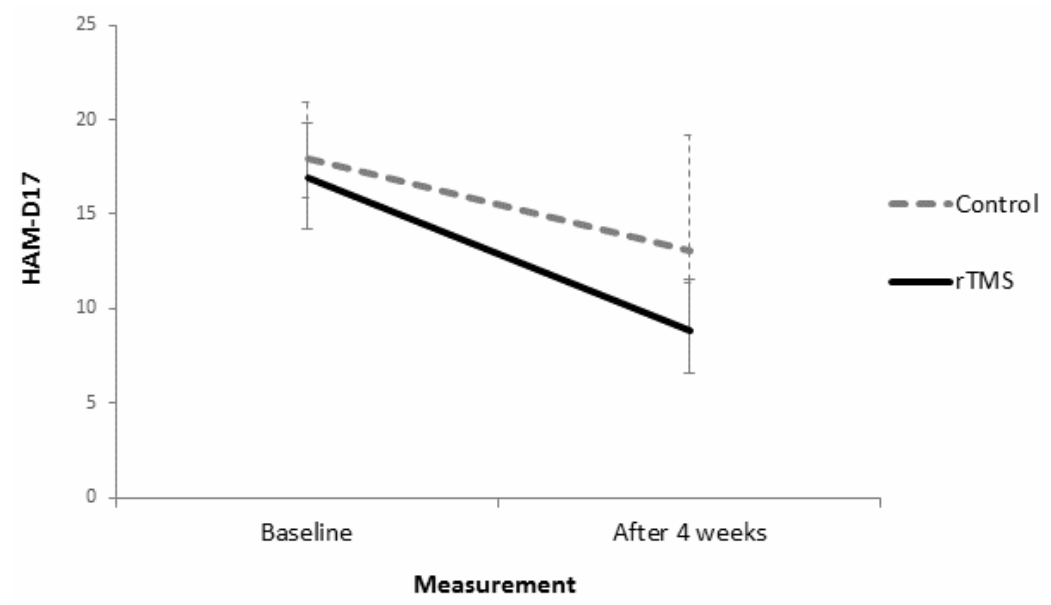

Figure 2. HAM-D17 at baseline and at $4^{\text {th }}$ week follow up; error bars represent $95 \%$ confidence intervals

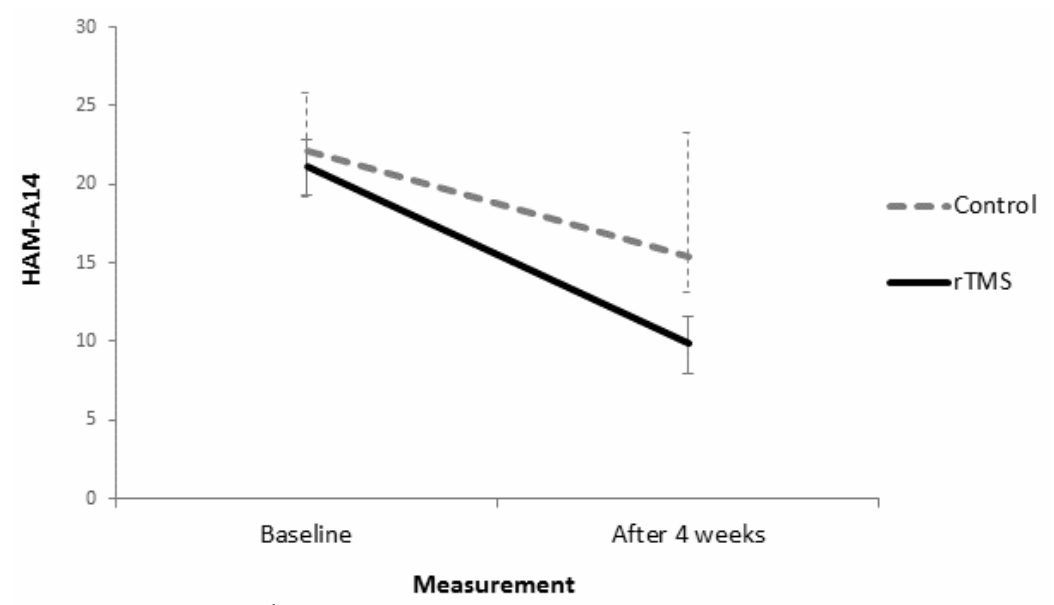

Figure 3. HAM-A14 at baseline and at $4^{\text {th }}$ week follow up; error bars represent $95 \%$ confidence intervals

\section{DISCUSSION}

Our study showed that HF rTMS was more effective and equally tolerable as the standard procedure in MDD in Croatian patients.

The efficacy of HF rTMS of the left DLPFC in depression is broadly documented (Lefaucher et al. 2014), although results from different studies vary with regard to the level of this efficacy (e.g., Loo et al. 1999, Rumi et al. 2005). In our study after four weeks of treatment with augmentative rTMS in MDD patients, a significant decrease $(48 \%)$ in scores on the Hamilton depression rating scale HAM-D (from 17 to 9) (Figure 2) was observed, as well as significant decrease $(53 \%)$ on HAM-A (from 21 to 10) (Figure 3). Although not statistically significant, we noticed indicative improvement in quality of life, particularly with regard to their psychological health (25\%). After 4 weeks of treatment, control group of patients with MDD showed decrease $(24 \%)$ in scores on the Hamilton depression rating scale HAM-D (from 18 to 13) and decrease $(30 \%)$ on HAM-A (from 22 to 15 ) (Figures 2 and 3). Patients in the group treated with augmentative rTMS compared to patients in control group have larger decrease in HAM-D 17: 48\% versus 24\%, and in HAM-A 14: $53 \%$ versus $30 \%$.
Although our study was not randomized and our control condition was not sham coil, the results are similar to first meta-analysis exploring the efficacy of augmentative rTMS for TRD (Liu et al. 2014). In that meta-analysis seven RCTs were included. Results from six RCTs showed that as a whole, 68/146 (46.6\%) and $15 / 84(22.1 \%)$ subjects in the active or sham rTMS groups were classified as responders, respectively. Also, in meta-analysis by Fitzgerald et al. (2016) which studied the rate of clinical response and remission, and the demographic and clinical predictors of response to rTMS from 11 clinical trials, remission rate was $31 \%$, and the rate of clinical response was $46 \%$. The results from our study showed that in a group of patients treated with rTMS in combination with medication 21/41 (51.2\%) were responders (a decrease in HAM-D scores of at least $50 \%)$ and $21 / 41(51.2 \%)$ remitters (scores in HAM-D of 7 or less).

Antidepressants are first line in the treatment of major depressive disorder, and all of our patients were treated with these drugs prior to TMS therapy. In our study, both groups received psychotropic medication in adequate and comparable doses but TMS group showed significantly greater decrease in HAM-D score HAM-A scores. Earlier studies have shown that rTMS hastens the response to antidepressant drugs in patients with major depressive 
disorder (Rossini et al. 2005). Also, after the failure of medications, rTMS significantly increases the effect of antidepressants (Liu et al. 2014). More specifically, rTMS accelerated the onset of action and augmented the response to amitriptyline (Rumi at al. 2005), and as an add-on strategy of clinical significance in combination with escitalopram in patients with major depression resistant to non-tricyclic antidepressants (Bretlau et al. 2008).

In this study, we observed some improvement in quality of life of patients, particularly in psychological health, but it was not statistically significant. Some other studies described statistically significant improvements in quality of life after four week treatment. Solvason et al. (2014) observed statistically significant improvement in both functional status and QOL ((SF-36) and (Q-LES-Q)) outcomes in patients treated with active TMS compared with sham TMS during the acute phase of the randomized, sham-controlled trial (Solvason et al. 2014). Janicak and colleagues (2013) informed of significant improvement in functional status on a broad range of mental health and physical health domains on the SF-36 following acute TMS treatment). Similarly, statistically significant improvement in patient-reported QOL was observed on all domains of the EQ-5D and on the General Health Perception and Health Index scores. Berlim et al. (2011) found significant increase in the WHOQOL BREF scores for the global, physical and psychological QOL domains from baseline to week four in naturalistic trial, on 15 TRD patients.

Several clinical trials have confirmed the safety of rTMS. Induction of seizure is the most severe adverse effect, but given the small number of cases of seizures caused by rTMS, it is clear that the risk is extremely low (Del Osso et al. 2011). More often there are minor side effects in the sense of discomfort during the stimulation, local pain, pain in the neck, transient hearing changes and headaches after stimulation (Del Osso et al. 2011). In our study only few patients receiving rTMS reported headache and more of them reported site discomfort and facial twitching. Patients did not discontinue study participation because of adverse events. Two patients in rTMS group and two patients in control group discontinued study participation because of personal reasons. During rTMS treatment occurred no seizures.

In large, naturalistic, observational follow up study, TMS demonstrated a sustained durability of effect over 12 months (Dunner et al. 2014). To assess long term effectiveness of rTMS therapy patients are currently participating in follow up study.

\section{Limitations of the study}

Our study had several limitations. First, we did not randomize participants into the intervention and control group. For this reason, we controlled only the endogenous confounding factors that were explicitly included and measured. Second, there were relatively large baseline differences between our two study groups. We tried to control this source of bias by multivariate analysis. Third, the outcome assessment was not done independently and blindly. The effect of this bias was most likely against the null hypothesis. Future studies with independent outcome assessment are needed in order to verify our findings.

\section{CONCLUSION}

It seems that augmentative treatment with HF rTMS is more effective on depression and anxiety symptoms than standard therapy in MDD with equal safety and tolerability. Randomized, controlled studies are required to verify this finding.

\section{Acknowledgements:}

Authors wish to acknowledge the work of all patients, physicians, and medical nurses who took part in the data collection.

The study was funded by Psychiatric Hospital Sveti Ivan, Zagreb, Croatia.

Conflict of interest: None to declare.

\section{Contribution of individual authors:}

Igor Filipcic was involved with study design, data collection, data interpretation, and manuscript preparation.

Zeljko Milovac participated in the writing and multiple edits of the manuscript drafts and data collection.

Strahimir Sucic, Tomislav Gajsak, Vjekoslav Aljinovic, Ivana Orgulan and Sandra Zecevic Penic participated in data collection.

Ivona Simunovic Filipcic and Ena Ivezic reviewed the manuscript drafts.

Zarko Bajic performed the statistical analyses and data interpretation

\section{References}

1. Bench CJ, Frackowiak RS \& Dolan RJ: Changes in regional cerebral blood flow on recovery from depression. Psychol Med 1995; 25:247-61.

2. Berlim MT, McGirr A, Beaulieu MM \& Turecki G: High frequency repetitive transcranial magnetic stimulation as an augmenting strategy in severe treatment-resistant major depression: A prospective 4-week naturalistic trial. J Affect Disord 2011; 130:312-17.

3. Berlim MT, van den Eynde F, Tovar-Perdomo $S$ \& Daskalakis ZJ: Response, remission and drop-out rates following high-frequency repetitive transcranial magnetic stimulation (rTMS) for treating major depression: a systematic review and meta-analysis of randomized, double-blind and sham controlled trials. Psychol Med 2014; 44: 225-39.

4. Bobo WV, Anglero GC, Jenkins G, Hall-Flavin DK, Weinshilboum $R$ \& Biernacka JM: Validation of the 17-item Hamilton Depression Rating Scale definition of response for adults with major depressive disorder using equipercentile linking to Clinical Global Impression scale ratings: analysis of Pharmacogenomic Research Network Antidepressant Medication Pharmacogenomic Study (PGRN-AMPS) data. Hum Psychopharmacol 2016 May; 31:185-92. 
5. Bretlau LG, Lunde M, Lindberg L, Unden M, Dissing $S \&$ Bech P: Repetitive transcranial magnetic stimulation (rTMS) in combination with escitalopram in patients with treatment-resistant major depression: a double-blind, randomised, sham controlled trial. Pharmacopsychiatry 2008; 41:41-7.

6. Cusin C, Yang H, Yeung A \& Fava M:Rating scales for depression. In Bear L \& Blais MA (eds): Handbook of Clinical Rating Scales and Assessment in Psychiatry and Mental Health, 7-36. Humana Press, 2009.

7. Dell'Osso B, Camuri G, Castellano F, Vecchi V, Benedetti $M$, Bortolussi $S$ et al: Meta review of metanalytic studies with repetitive transcranial magnetic stimulation (rTMS) for the treatment of major depression. Clin Pract Epidemiol Ment Health 2011; 7:167-77.

8. Dunner DL, Aaronson ST, Sackeim HA, Janicak $P$, Carpenter L, Boyadjis $T$ et al: A multisite, naturalistic, observational study of transcranial magnetic stimulation for patients with pharmacoresistant major depressive disorder: durability of benefit over a 1-year follow-up period. J Clin Psychiatry 2014; 75:1394-401.

9. Fitzgerald PB, Hoy KE, Anderson RJ \& Daskalakis ZJ: A study of the pattern of response to rTMS treatment in depression. Depress Anxiety 2016; 33:746-53.

10. Gaynes BN, Lloyd SW, Lux L, Gartlehner G, Hansen RA, Brode $S$ et al: Repetitive transcranial magnetic stimulation for treatment-resistant depression: a systematic review and meta-analysis. J Clin Psychiatry. 2014; 75:477-89.

11. George MS, Lisanby SH, Avery D, McDonald WM, Durkalski V, Pavlicova $M$ et al: Daily left prefrontal transcranial magnetic stimulation therapy for major depressive disorder: a sham-controlled randomized trial. Arch Gen Psychiatry 2010; 67:507-16.

12. George MS, Wassermann EM, Kimbrell TA, Little JT, Williams WE, Danielson AL et al: Mood improvement following daily left prefrontal repetitive transcranial magnetic stimulation in patients with depression: a placebo-controlled crossover trial. Am J Psychiatry 1997; 154:1752-6.

13. Hamilton MA: Rating scale for depression. J Neurol Neurosurg Psychiatry 1960; 23:56-62.

14. Hamilton M: The assessment of anxiety states by rating. Br J Med Psychol 1959; 32:50-5.

15. Janicak PG, Dunner DL, Aaronson ST, Linda L, Carpenter T, Boyadjis TA et al: Transcranial magnetic stimulation (TMS) for major depression: a multisite, naturalistic, observational study of quality of life outcome measures in clinical practice. CNS Spectrums 2013; 18:322-32.

16. Kennedy SH, Javanmard $M$ \& Vaccarino FJ: A review of functional neuroimaging in mood disorders: positron emission tomography and depression. Can J Psychiatry 1997; 42:467-75.

17. Kyle PR, Lemming OM, Timmerby N, Søndergaard S, Andreasson $K \&$ Bech P: The Validity of the Different Versions of the Hamilton Depression Scale in Separating Remission Rates of Placebo and Antidepressants in Clinical Trials of Major Depression. J Clin Psychopharmacol 2016; 36:453-6.
18. Lan MJ, Chhetry BT, Liston C, Mann JJ \& Dubin M: Transcranial Magnetic Stimulation of Left Dorsolateral Prefrontal Cortex Induces Brain Morphological Changes in Regions Associated with a Treatment Resistant Major Depressive Episode: An Exploratory Analysis. Brain Stimul 2016; 9:577-83.

19. Lefaucheur JP, André-Obadia N, Antal A, Ayache SS, Baeken C, Benninger DH et al: Evidence-based guidelines on the therapeutic use of repetitive transcranial magnetic stimulation (rTMS). Clin Neurophysiol 2014; 125:2150-206.

20. Liu B, Zhang Y, Zhang L \& Li L: Repetitive transcranial magnetic stimulation as an augmentative strategy for treatment-resistant depression, a meta-analysis of randomized, double-blind and sham-controlled study. BMC Psychiatry 2014; 14:342.

21. Loo C, Mitchell P, Sachdev P, McDarmont B, Parker G \& Gandevia S: Double-blind controlled investigation of transcranial magnetic stimulation for the treatment of resistant major depression. Am J Psychiatry 1999; 156:946-8.

22. Mihaljević Peleš A \& Šagud M: Psychopharmacology guidelines for treating depression. Medix 2013, 106:151-5.

23. O'Reardon JP, Solvason HB, Janicak $P G$, Sampson $S$, Isenberg KE, Nahas $Z$, et al: Efficacy and safety of transcranial magnetic stimulation in the acute treatment of major depression: a multisite randomized controlled trial. Biol Psychiatry 2007; 62:1208-16.

24. Paus T, Castro-Alamancos MA \& Petrides M: Corticocortical connectivity of the human mid-dorsolateral frontal cortex and its modulation by repetitive transcranial magnetic stimulation. Eur J Neurosci 2001; 14:1405-11.

25. Rumi DO, Gattaz WF, Rigonatti SP, Rosa MA, Fregni F, Rosa MO et al: Transcranial magnetic stimulation accelerates the antidepressant effect of amitriptyline in severe depression: a double-blind placebo-controlled study. Biol Psychiatry 2005; 57:162-6.

26. Rush AJ, Trivedi $M \&$ Fava M: Depression, IV: $S T A R^{*} D$ treatment trial for depression. Am J Psychiatry 2003; 160:237.

27. Solvason HB, Husain M, Fitzgerald PB, Rosenquist $P$, McCall WV, Kimball J et al: Improvement in Quality of Life With Left Prefrontal Transcranial Magnetic Stimulation in Patients With Pharmacoresistant Major Depression: Acute and Six Month Outcomes. Brain Stimulation 2014; 7:219-25.

28. Thomson E: Hamilton Rating Scale for Anxiety (HAM-A). Occup Med (Lond) 2015; 65:601.

29. World Health Organization: Study protocol for the World Health Organization project to develop a Quality of Life assessment instrument (WHOQOL). Qual Life Res 1993; 2:153-9.

30. World Health Organization: WHOQOL-BREF: Introduction, administration, scoring and generic version of the assessment. WHO, Geneva, 1996.

31. World Health Organization 2016. Depression: Fact Sheet Number 369. Available from: http://www.who.int/mediacentre/factsheets/fs369/en/

32. World Medical Association: World Medical Association Declaration of Helsinki: ethical principles for medical research involving human subjects. JAMA 2013; 310:2191-4.

\section{Correspondence:}

Igor Filipcic, $M D, P h D$

Psychiatric Hospital "Sveti Ivan"

Jankomir 11, pp68, HR-10 090 Zagreb, Croatia

E-mail: igor.filipcic@pbsvi.hr 